\title{
Airway Remodeling in Asthma: Evaluation in 5 Consecutive Bronchial Generations by Using High-Resolution Computed Tomography
}

\author{
Dan Jiang MM, Zhimin Wang MM, Nan Yu PhD, Cong Shen MM, Lei Deng MM, and \\ Youmin Guo PhD
}

BACKGROUND: Airway remodeling is a characteristic structural change that occurs extensively in the airways of patients with asthma. The change can be evaluated by measuring airway dimensions by using high-resolution computed tomography. This study aimed to explore the variation trends of airway dimensions from the second- to sixth-generation bronchi in subjects with asthma. METHODS: Sixty subjects with asthma and 40 healthy controls underwent high-resolution computed tomography. The right upper lobe apical segmental bronchus, right lower lobe posterior basal segmental bronchus, left upper lobe apicoposterior segmental bronchus, and the left lower lobe posterior basal segmental bronchus were identified on computed tomography images. The luminal area, wall area, and wall area percentage of each bronchus were measured from the second (lobar) to the sixth generation. The variation trends of these indices (luminal area, wall area, and wall area percentage) were then compared between the subjects with asthma and the healthy controls. RESULTS: From the proximal to the distal airway, the luminal area and wall area decreased gradually, whereas the wall area percentage increased gradually in all the participants; the difference between the participants with asthma and the healthy controls in the luminal area, wall area, and wall area percentage increased gradually from the second (lobar) to the sixth generation; there was a significant difference between the participants with asthma and the healthy controls in the right lower lobe posterior basal segmental bronchus, left upper lobe apicoposterior segmental bronchus, and left lower lobe posterior basal segmental bronchus wall area percentage $(P=.001, .01$ and .001 , respectively) but not in the right upper lobe apical segmental bronchus wall area percentage $(P=\mathbf{. 0 5 0})$. CONCLUSIONS: Airway remodeling in asthma was more prominent in distal airways and at lower lobe bronchi. Key words: asthma; airway remodeling; luminal area; wall area; wall area percentage; high-resolution computed tomography. [Respir Care 2018;63(11):1399-1406.

(c) 2018 Daedalus Enterprises]

\section{Introduction}

Asthma is a common chronic respiratory disease, usually characterized by chronic airway inflammation. Char-

\footnotetext{
Ms Jiang, Mr Wang, Dr Yu, Ms Shen, Ms Deng, and Dr Guo are affiliated with Department of Radiology, the First Affiliated Hospital of Xi' an Jiaotong University, Xi' an, Shanxi, China. Ms Jiang and Mr Wang are affiliated with Department of Radiology, Gansu Provincial Hospital, Lanzhou, Gansu, China.
}

This work was supported by funding from the Public Science and Technology research funds of China (201402013). acteristic structural changes, often described as airway remodeling, are observed in the airways of patients with asthma. ${ }^{1}$ Results from surgically resected lung tissue, autopsy lung specimens, and transbronchial biopsy speci-

\footnotetext{
The authors have disclosed no conflicts of interest.
}

Correspondence: Youmin Guo PhD, Department of Radiology, The First Affiliated Hospital of Xi'an Jiaotong University, Yanta Western Road, Xi'an, Shanxi, China, 710061. E-mail: guoyoumin163@sina.com.

DOI: $10.4187 /$ respcare. 06050 
mens indicate that airway remodeling (subepithelial fibrosis, increased airway smooth muscle, increased blood vessels in airway walls, and mucus hypersecretion) occurs in both central and peripheral airways, and can result in bronchial wall thickening. ${ }^{2-9}$ Further research indicates that airway remodeling is related to the severity of the disease and may result in relatively irreversible narrowing of the airways. ${ }^{10,11}$

Many studies have assessed the structural changes that occur in asthma by using high-resolution computed tomography $(\mathrm{CT}),{ }^{12-17}$ in which airway-dimension indices, such as the luminal area, wall area, and wall area percentage, of a selected segmental bronchus are measured by using manual or automated techniques. Some of these studies demonstrated that the airway walls of subjects with asthma are thicker than those of healthy subjects and that airway wall thickening is related to air-flow obstruction and disease severity. ${ }^{12-17}$ However, in previous studies, ${ }^{12-17}$ one measurement point that was selected in a bronchus for analysis does not reflect the range and the variation trends of structural changes in airways of patients with asthma.

In the present study, dimension indices (luminal area, wall area, and wall area percentage) of selected bronchi were measured from the second (lobar) to the sixth generation and were compared between the subjects with asthma and the healthy controls. We intended to investigate 2 questions: (1) how do the bronchial dimensions vary from the second- to sixth-generation airways, and (2) do the variation trends differ between the subjects with asthma and the healthy controls?

\section{Methods}

\section{Participants}

All participants were recruited from our hospital between March 2015 and March 2016. Subjects who met the following inclusion criteria were recruited: (1) asthma diagnosed by a respiratory physician based on the history of characteristic symptom patterns and evidence of variable air-flow limitation, (2) adults (ages $\geq 18 \mathrm{y}$ ), (3) lifetime nonsmokers, (4) no other respiratory diseases (according to medical history and related examinations). The severity of asthma was assessed according to the Global Initiative for Asthma guidelines, ${ }^{1}$ based on the level of treatment required to control symptoms and exacerbations. Spirometry was performed within $1-2 \mathrm{~d}$ of an high-resolution $\mathrm{CT}$, and, on the same day, blood samples were taken for measurements of the serum eosinophil and neutrophil percentages. The healthy controls were recruited from the health examination center of our hospital. All were free from respiratory symptoms and respiratory diseases and were lifetime nonsmokers. The study was approved by the eth-

\section{QUICK LOOK}

\section{Current knowledge}

Airway remodeling is a characteristic structural change that occurs extensively in the airways of patients with asthma. The change can be evaluated by measuring airway dimensions by using high-resolution computed tomography.

\section{What this paper contributes to our knowledge}

From the proximal to the distal airway, the luminal area, and wall area decreased gradually, whereas the wall area percentage increased gradually in all the participants; the difference between the subjects with asthma and the healthy controls in the luminal area, wall area, and wall area percentage increased gradually from the second (lobar) to sixth generation. Airway remodeling in asthma was more prominent in distal airways and at lower lobe bronchi.

ics committee of our institution, and written informed consent was obtained from all the participants.

\section{CT and Data Analysis}

Volumetric whole-lung scans were performed by using a multidetector CT scanner (GE Light Speed VCT; General Electric, Milwaukee, Wisconsin) with the following parameters: collimation, $64 \times 0.625 \mathrm{~mm} ; 120 \mathrm{kVp}$; $160 \mathrm{mAs}$; rotation time, $0.6 \mathrm{~s}$; and pitch, 0.984. All the participants were scanned while they were in the supine position at full-inspiration (inspiratory breath-hold was rehearsed before the CT) and with arms held overhead. Images were reconstructed by using a high-spatial frequency algorithm with a slice thickness of $1.25 \mathrm{~mm}$ and an interval of $1.25 \mathrm{~mm}$. A 3-dimensional bronchial skeleton was then automatically reconstructed by using the volumetric data. The right upper lobe apical segmental bronchus, right lower lobe posterior basal segmental bronchus, left upper lobe apicoposterior segmental bronchus, and left lower lobe posterior basal segmental bronchus were selected and automatically converted to curved multiplanar reconstruction images (we selected these bronchi because they are relatively perpendicular to CT images and less affected by cardiac motion artifacts).

The second- (lobar) to sixth-generation airways of each bronchus were identified and analyzed (the right lower lobe bronchus is the third generation, thus, for right lower lobe posterior basal segmental bronchus, the third to seventh generations were identified and analyzed). The values of the luminal area, wall area, and wall area percentage of 
each generation were calculated automatically and recorded (the wall area and wall area percentage were derived from the total area and luminal area as follows: wall area $=$ total area - luminal area; wall area percentage $=$ wall area/total area $\times 100 \%)$. The processes of bronchial selection, identification, and calculation were carefully inspected by using the longitudinal and short-axis images simultaneously by one author (DJ) who was blinded to the participants' information.

\section{Statistical Analysis}

Statistical analyses were performed with SPSS version 17.0 (SPSS, Chicago, Illinois). Parametric data were expressed as mean $\pm \mathrm{SD}$. Comparisons of age and body mass index between the 2 groups were performed by using

Table 1. Characteristics of the Subjects

\begin{tabular}{|c|c|c|c|}
\hline & $\begin{array}{l}\text { Asthma Group } \\
(n=60)\end{array}$ & $\begin{array}{l}\text { Control Group } \\
\quad(n=40)\end{array}$ & $P$ \\
\hline Age, y & $50.17 \pm 14.59$ & $49.00 \pm 6.45$ & $.59 *$ \\
\hline Men/women, $n$ & $24 / 36$ & $18 / 22$ & $.62 \dagger$ \\
\hline Body mass index, $\mathrm{kg} / \mathrm{m}^{2}$ & $24.65 \pm 3.54$ & $24.78 \pm 3.74$ & $.87 *$ \\
\hline Disease duration, y & $9.74 \pm 10.64$ & & \\
\hline $\begin{array}{l}\text { Disease severity } \\
\quad \text { mild/moderate/severe, } n\end{array}$ & $22 / 18 / 20$ & & \\
\hline $\mathrm{FEV}_{1}, \%$ predicted & $70.11 \pm 22.61$ & & \\
\hline $\mathrm{FEV}_{1} \mathrm{FVC}, \%$ & $70.45 \pm 13.72$ & & \\
\hline Serum eosinophils, $\%$ & $3.66 \pm 4.58$ & & \\
\hline Serum neutrophils, $\%$ & $64.54 \pm 12.29$ & & \\
\hline $\begin{array}{l}\text { Data are expressed as mean SD. } \\
* \text { Unpaired } t \text { test. } \\
\dagger \text { Chi-square test. }\end{array}$ & & & \\
\hline
\end{tabular}

the unpaired $t$ test or chi-square test. Comparisons of the luminal area, wall area, and wall area percentage between the patients with asthma and the healthy controls were performed by using multivariate repeated-measures analysis of variance; the Mauchly test of sphericity was used to judge whether there were relationships among the repeatedly measured data; multivariate analysis of variance was performed if $P>.05$, otherwise Greenhouse-Geisser corrected results were taken. In the subjects with asthma, analysis of variance was used for comparison of the wall area percentage between and among groups with different clinical characteristics (age, sex, body mass index, disease duration, disease severity, $\mathrm{FEV}_{1} \%$ predicted, $\mathrm{FVC}, \mathrm{FEV}_{1}$ : FVC (\%), serum eosinophils and serum neutrophils). $P<.05$ was considered to indicate statistical significance.

\section{Results}

Sixty subjects with asthma and 40 healthy controls were included in the study. The characteristics of all the participants are shown in Table 1. Among the 60 subjects with asthma, 22 were classified as having mild, 18 were classified as having moderate, and 20 were classified as having severe asthma according to the Global Initiative for Asthma Guidelines. ${ }^{1}$ No significant differences were found in age, sex, or body mass index between the subjects with asthma and the healthy controls. The second (lobar) to sixth generations of the right upper lobe apical segmental bronchus, right lower lobe posterior basal segmental bronchus (third to seventh generations), left upper lobe apicoposterior segmental bronchus, and left lower lobe posterior basal segmental bronchus were identified in 56, 58, 56, and 57 subjects with asthma, respectively, and these bron-

Table 2. Comparison of the Luminal Area, Wall Area, and Wall Area Percentage of RB10

\begin{tabular}{|c|c|c|c|c|c|c|c|}
\hline Area & $n$ & G3 & G4 & G5 & G6 & G7 & $P^{*}$ \\
\hline \multicolumn{8}{|c|}{ Luminal area, $\mathrm{mm}^{2}$} \\
\hline Asthma & 58 & $39.54 \pm 10.70$ & $21.27 \pm 7.81$ & $11.73 \pm 4.67$ & $8.65 \pm 2.95$ & $6.07 \pm 2.49$ & \\
\hline Control & 40 & $41.83 \pm 10.52$ & $24.71 \pm 8.36$ & $14.50 \pm 5.64$ & $12.01 \pm 4.11$ & $8.30 \pm 4.34$ & .008 \\
\hline$P \dagger$ & & .30 & .040 & .009 & $<.001$ & .002 & \\
\hline \multicolumn{8}{|c|}{ Wall area, $\mathrm{mm}^{2}$} \\
\hline Asthma & 58 & $26.91 \pm 5.89$ & $20.58 \pm 6.14$ & $15.67 \pm 3.59$ & $14.02 \pm 2.95$ & $12.65 \pm 2.23$ & \\
\hline Control & 40 & $25.09 \pm 5.26$ & $18.50 \pm 4.58$ & $14.36 \pm 3.43$ & $12.78 \pm 2.15$ & $11.56 \pm 2.30$ & .033 \\
\hline$P \dagger$ & & .12 & .07 & .08 & .03 & .02 & \\
\hline \multicolumn{8}{|l|}{ Wall area $\%$} \\
\hline Asthma & 58 & $0.41 \pm 0.10$ & $0.49 \pm 0.12$ & $0.58 \pm 0.13$ & $0.62 \pm 0.09$ & $0.69 \pm 0.10$ & \\
\hline Control & 40 & $0.38 \pm 0.08$ & $0.45 \pm 0.11$ & $0.51 \pm 0.11$ & $0.53 \pm 0.12$ & $0.60 \pm 0.14$ & .001 \\
\hline$P \dagger$ & & .12 & .056 & .007 & $<.001$ & $<.001$ & \\
\hline \multicolumn{8}{|c|}{$\begin{array}{l}\text { Data are expressed as mean SD. } \\
\text { * Multivariate repeated-measures analysis of variance. } \\
\dagger \text { Multivariate analysis of variance. } \\
\mathrm{G}=\text { airway generation } \\
\text { RB10 = right lower lobe posterior basal segmental bronchus }\end{array}$} \\
\hline
\end{tabular}


chi were identified in all 40 healthy controls. In both the subjects with asthma and in the healthy controls, the luminal area and the wall area of the right upper lobe apical segmental bronchus, right lower lobe posterior basal segmental bronchus, left upper lobe apicoposterior segmental bronchus, and left lower lobe posterior basal segmental bronchus decreased gradually from the second (lobar) to the sixth generation, whereas the wall area percentage of these bronchi increased gradually.

There were significant differences between the subjects with asthma and the healthy controls in the luminal area (right upper lobe apical segmental bronchus, $P=.032$; right lower lobe posterior basal segmental bronchus, $P=.008$; left upper lobe apicoposterior segmental bronchus $P=.02$; left lower lobe posterior basal segmental bronchus, $P=.01$ ) and the wall area (right upper lobe apical segmental bronchus, $P=.048$; right lower lobe posterior basal segmental bronchus, $P=.033$; left upper lobe apicoposterior segmental bronchus, $P=.042$; and left lower lobe posterior basal segmental bronchus, $P=.039$ ); compared with the healthy controls, the luminal area was smaller and the wall area was larger in the asthma group. The luminal area and wall area values from the third to seventh generation of the right lower lobe posterior basal segmental bronchus are shown in Table 2; their variation trends are shown in Figure 1.

There was a significant difference between the subjects with asthma and the healthy controls in the right lower lobe posterior basal segmental bronchus, left upper lobe apicoposterior segmental bronchus, and left lower lobe posterior basal segmental bronchus wall area percentage $(P=.001, P=.01$, and $P<.001$, respectively) but not in the right upper lobe apical segmental bronchus wall area percentage $(P=.050)$. The wall area percentage in the subjects with asthma was larger than that of the healthy controls. Further comparison of the wall area percentage between the subjects with asthma and the healthy controls at each generation showed significant differences in the right upper lobe apical segmental bronchus at the sixth generation $(P=.002)$, in the left upper lobe apicoposterior segmental bronchus at the fifth and sixth generations $(P=.006$ and $P=.009$, respectively), in the right lower lobe posterior basal segmental bronchus at the fifth, sixth, and seventh generations $(P=.007, P<.001$, and $P<.001$, respectively), and in the left lower lobe posterior basal segmental bronchus at the fourth, fifth, and sixth generations $(P=.066, P<.001$, and $P=.001$ respectively). The values and the variation trends of the wall area percentage from the second (lobar) to sixth generation of the right upper lobe apical segmental bronchus, right lower lobe posterior basal segmental bronchus (third to seventh generation), left upper lobe apicoposterior segmental bronchus, and left lower lobe posterior basal segmental bronchus are shown in Table 3 and Figure 2.
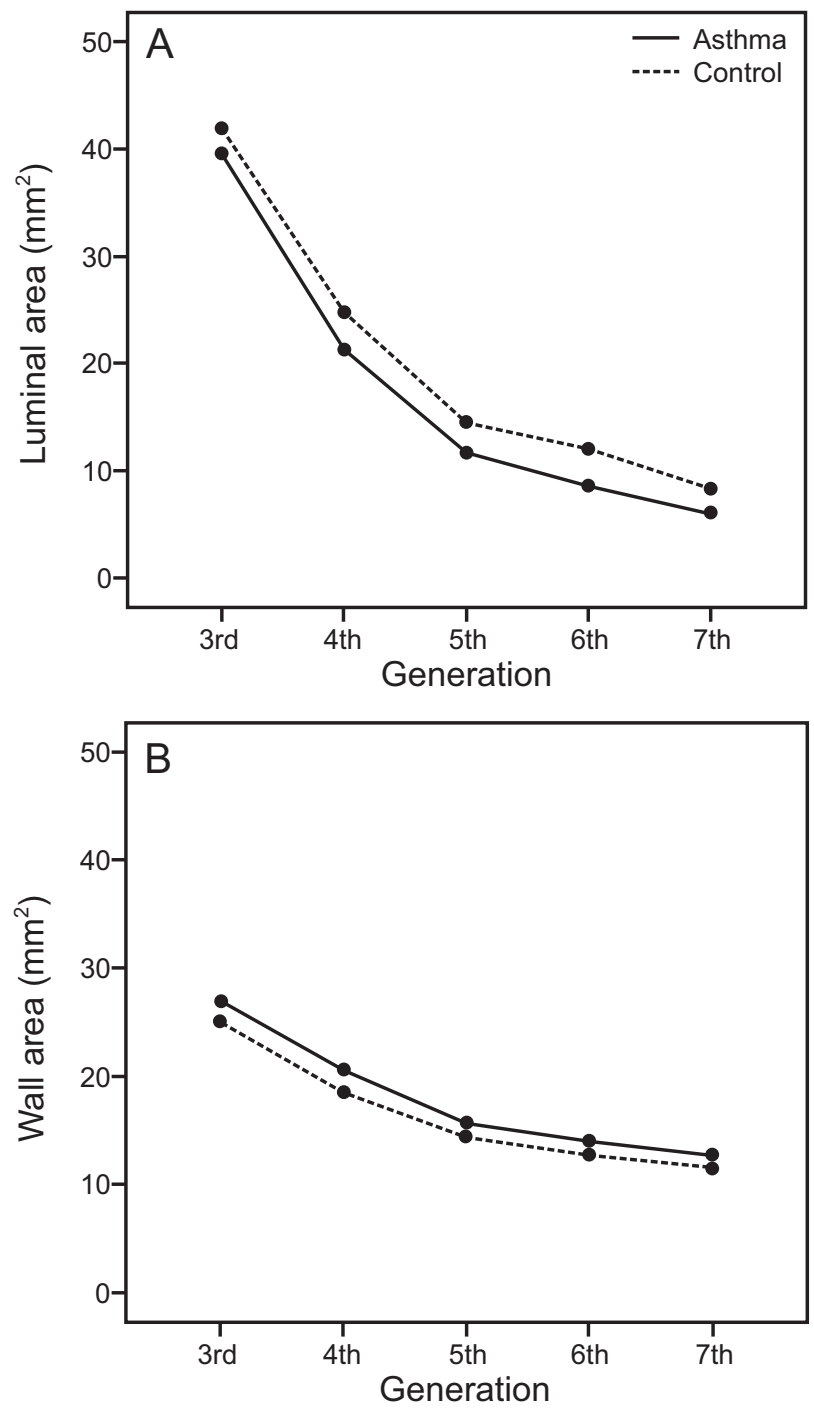

Fig. 1. The variation trends of the luminal area (LA) $(A)$ and the wall area (WA) (B) of the right lower lobe posterior basal segmental bronchus (RB10). The LA was smaller and the WA was larger in the subjects with asthma; from the third- (the right lower lobe bronchus) to the seventh-generation, LA and WA decreased gradually.

In the 58 subjects with asthma whose right lower lobe posterior basal segmental bronchus was identified from the third (inferior lobe of right lung) to seventh generation by using age, sex, body mass index, disease duration, disease severity, $\mathrm{FEV}_{1} \%$ predicted, $\mathrm{FVC}, \mathrm{FEV}_{1}: \mathrm{FVC}(\%)$, serum eosinophils (\%), and serum neutrophils (\%) as between-subjects factor respectively, significant differences were identified in the wall area percentage between normal and low $\mathrm{FEV}_{1}: \mathrm{FVC}(\%)$ groups $(P=.01)$ and among different severity groups $(P=.047)$, the value of the wall area percentage of the right lower lobe posterior basal segmental bronchus is shown in Table 4. 
Table 3. Comparison of RB1, RB10, LB1+2, and LB10 Wall Area Percentages

\begin{tabular}{|c|c|c|c|c|c|c|c|}
\hline & $n$ & G2 & G3 & G4 & G5 & G6 & $P^{*}$ \\
\hline \multicolumn{8}{|l|}{ RB1 } \\
\hline Asthma & 56 & $0.36 \pm 0.03$ & $0.55 \pm 0.10$ & $0.63 \pm 0.06$ & $0.75 \pm 0.08$ & $0.81 \pm 0.05$ & \\
\hline Control & 40 & $0.37 \pm 0.03$ & $0.52 \pm 0.17$ & $0.61 \pm 0.04$ & $0.72 \pm 0.03$ & $0.77 \pm 0.04$ & .050 \\
\hline$P \dagger$ & & .10 & .40 & .22 & .058 & .002 & \\
\hline \multicolumn{8}{|l|}{ RB10 } \\
\hline Asthma & 58 & $0.41 \pm 0.10$ & $0.49 \pm 0.12$ & $0.58 \pm 0.13$ & $0.62 \pm 0.09$ & $0.69 \pm 0.10$ & \\
\hline Control & 40 & $0.38 \pm 0.08$ & $0.45 \pm 0.11$ & $0.51 \pm 0.11$ & $0,53 \pm 0.12$ & $0.60 \pm 0.14$ & .001 \\
\hline$P \dagger$ & & .12 & .056 & .007 & $<.001$ & $<.001$ & \\
\hline \multicolumn{8}{|l|}{$\mathrm{LB} 1+2$} \\
\hline Asthma & 56 & $0.35 \pm 0.03$ & $0.47 \pm 0.06$ & $0.62 \pm 0.08$ & $0.71 \pm 0.05$ & $0.77 \pm 0.04$ & \\
\hline Control & 40 & $0.36 \pm 0.03$ & $0.46 \pm 0.06$ & $0.60 \pm 0.05$ & $0.68 \pm 0.05$ & $0.75 \pm 0.04$ & .01 \\
\hline$P \dagger$ & & .25 & .19 & .09 & .006 & .009 & \\
\hline \multicolumn{8}{|l|}{ LB10 } \\
\hline Asthma & 57 & $0.38 \pm 0.06$ & $0.45 \pm 0.06$ & $0.56 \pm 0.08$ & $0.64 \pm 0.06$ & $0.71 \pm 0.05$ & \\
\hline Control & 40 & $0.37 \pm 0.03$ & $0.43 \pm 0.03$ & $0.54 \pm 0.06$ & $0.60 \pm 0.05$ & $0.67 \pm 0.05$ & $<.001$ \\
\hline$P \dagger$ & & .14 & .07 & .066 & .002 & $<.001$ & \\
\hline $\begin{array}{l}\text { Data are expres } \\
* \text { Multivariate } \\
\dagger \text { Multivariate } \\
\text { RB1 = right u } \\
\text { RB10 = right } \\
\text { LB1 } 1=\text { left } \\
\text { LB10 = left lo } \\
\text { G = airway ge }\end{array}$ & $\begin{array}{l}\text { an } \pm \\
\text { eeasur } \\
\text { variar } \\
\text { apical } \\
\text { poste } \\
\text { apic } \\
\text { osteri }\end{array}$ & $\begin{array}{l}\text { of variance. } \\
\text { bronchus } \\
\text { egmental bronchus } \\
\text { egmental bronchus } \\
\text { gmental bronchus }\end{array}$ & & & & & \\
\hline
\end{tabular}

\section{Discussion}

In this study, we measured airway dimension indices of 4 bronchi from the second (lobar) to sixth generation by using high-resolution CT and compared them between the subjects with asthma and the healthy controls. We found that, in all the participants, from the second (lobar) to sixth generation, the luminal area and the wall area decreased gradually, whereas the wall area percentage increased gradually. The subjects with asthma had a smaller luminal area, larger wall area, and larger wall area percentage compared with the healthy controls. The differences between the 2 groups in the wall area, luminal area, and wall area percentage were more prominent at the distal bronchi than they were at the proximal bronchi.

Several studies that researched proximal airway remodeling by using high-resolution CT reported various results of airway dimensions. ${ }^{12,13,18,19}$ These studies measured the selected bronchi only at one generation; however, asthma is a heterogeneous disease that involves both central and peripheral airways, thus evaluation of one generation cannot fully reflect the changes in airway dimension, which may explain the variability in airway dimensions reported by previous studies. ${ }^{12,13,18,19}$ Furthermore, our study demonstrated that, in the subjects with asthma, the larger wall area percentage should not be considered simply as the result of airway wall thickening: at the proximal airways, the larger wall area percentage was mainly derived from the smaller luminal area, which was consistent with the findings of Gupta et al ${ }^{13}$; at the distal airways, the larger wall area percentage was a common outcome of the smaller luminal area and the larger wall area. Therefore, the wall area percentage should be considered as an integrated indicator of airway structural changes both in the wall and the lumen.

The present study also revealed that the differences in the luminal area, wall area, and wall area percentage between the subjects with asthma and the healthy controls tended to increase from the proximal to the distal airways. Recently, interest in the role of small airways in asthma has increased. However technical limitations have hampered the direct measurement of most small airways, and the assessment of small airway involvement remains a challenge. ${ }^{5,6,20-22}$ Here, we sequentially measured airway dimensions from the second (lobar) to sixth generation of 4 bronchi and provided the variation trends of airway dimensions, which is helpful to predict small-airway involvement. First, parts of the sixth-generation bronchi (the seventh generation in the right lower lobe posterior basal segmental bronchus) measured in the study were $<2 \mathrm{~mm}$ in diameter and belonged to a small airway; second, from the proximal to the distal airways, the increasing differences between the subjects with asthma and the healthy controls indicated that similar but more-severe structural 

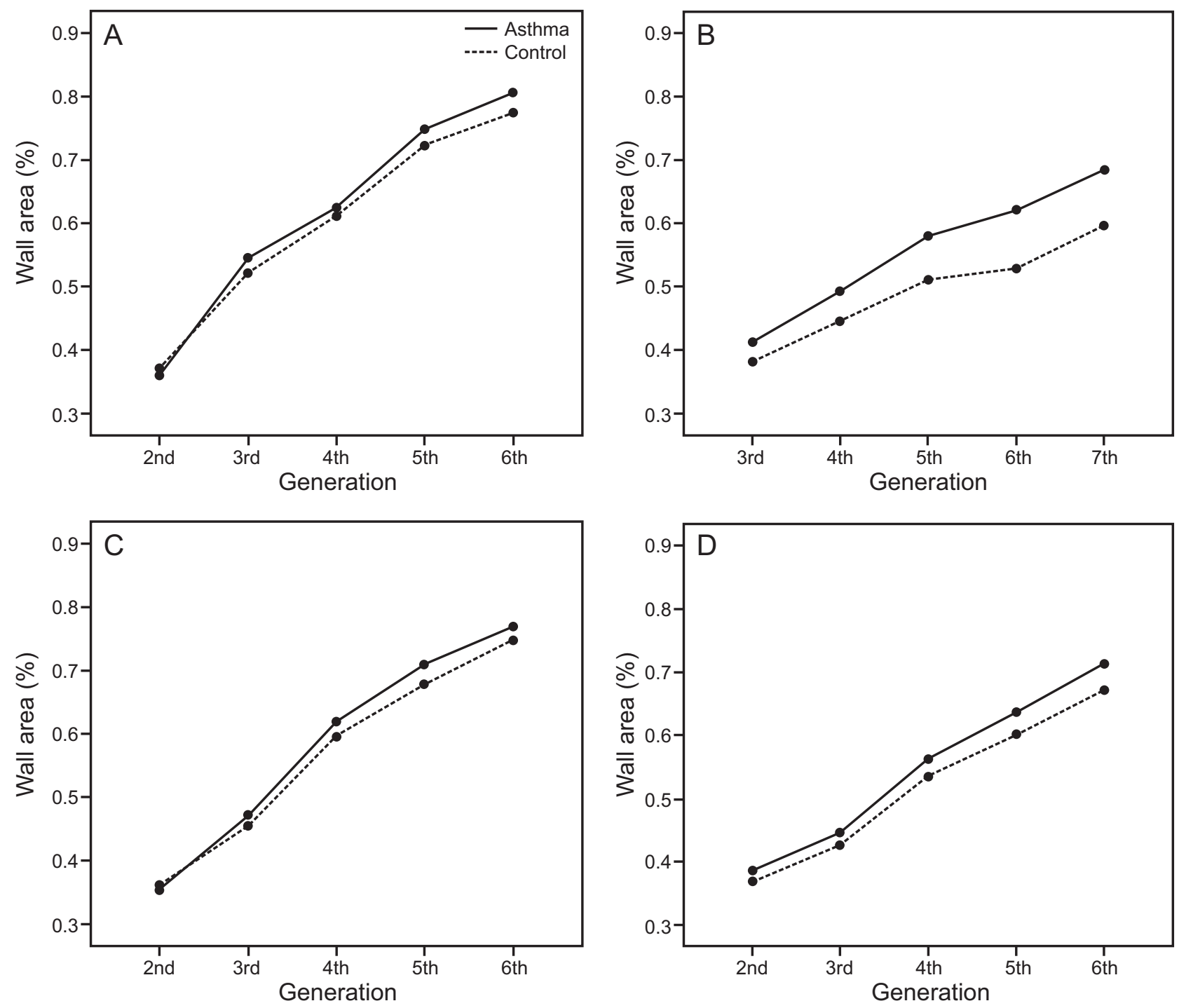

Fig. 2. The variation trends of the wall area percentage (WA\%) of the right upper lobe apical segmental bronchus (RB1) (A), the left upper lobe apicoposterior segmental bronchus $(\mathrm{LB} 1+2)(\mathrm{B})$, the right lower lobe posterior basal segmental bronchus (RB10) (C), and the left lower lobe posterior basal segmental bronchus (LB10) (D). WA\% was larger in asthma group; from the second- (lobar) to sixth-generation WA\% increased gradually.

Table 4. Comparison of the Wall Area Percentage of RB10 in Different FEV 1 :FVC and Severity Groups of Subjects With Asthma

\begin{tabular}{|c|c|c|c|c|c|c|c|}
\hline Parameter & $n$ & G3 & G4 & G5 & G6 & G7 & $P^{*}$ \\
\hline \multicolumn{8}{|l|}{$\mathrm{FEV}_{1}: \mathrm{FVC}$} \\
\hline Normal & 24 & $0.39 \pm 0.07$ & $0.47 \pm 0.12$ & $0.55 \pm 0.10$ & $0.59 \pm 0.08$ & $0.62 \pm 0.08$ & \\
\hline Low & 34 & $0.42 \pm 0.10$ & $0.52 \pm 0.13$ & $0.60 \pm 0.15$ & $0.65 \pm 0.09$ & $0.73 \pm 0.09$ & .01 \\
\hline \multicolumn{8}{|l|}{ Severity } \\
\hline Mild & 22 & $0.40 \pm 0.07$ & $0.48 \pm 0.10$ & $0.56 \pm 0.09$ & $0.59 \pm 0.08$ & $0.62 \pm 0.08$ & \\
\hline Moderate & 18 & $0.41 \pm 0.11$ & $0.50 \pm 0.14$ & $0.58 \pm 0.16$ & $0.62 \pm 0.10$ & $0.70 \pm 0.08$ & \\
\hline Severe & 18 & $0.43 \pm 0.10$ & $0.52 \pm 0.14$ & $0.62 \pm 0.1$ & $0.67 \pm 0.09$ & $0.75 \pm 0.09$ & .047 \\
\hline \multicolumn{8}{|c|}{$\begin{array}{l}\text { Data are expressed as mean } \pm \text { SD. } \\
\text { * Multivariate repeated-measures analysis of variance. } \\
\text { RB } 10 \text { = right lower lobe posterior basal segmental bronchus } \\
\mathrm{G}=\text { airway generation }\end{array}$} \\
\hline
\end{tabular}


changes occurred in the distal airways. Our findings might provide a new way to predict small airway involvement in asthma and help to identify subjects who might benefit from the therapy by using the extrafine particles to target the small airways.

Our findings showed that the differences in wall area percentage between the subjects with asthma and the healthy controls were more prominent at lower lobe bronchi than they were at upper lobe bronchi. Among the 4bronchi we studied, the right upper lobe apical segmental bronchus was the only one in which no significant difference was found in the wall area percentage between the subjects with asthma and the healthy controls. A similar result was reported by Gupta et $\mathrm{al}^{13}$ when they analyzed the percentage wall volume of the left upper lobe apicoposterior segmental bronchus. The heterogeneous distribution was found in some patients with asthma. ${ }^{23,24}$ In a study in subjects with stable asthma, Ueda et $\mathrm{al}^{20}$ found that greater degrees of air trapping were consistently observed at lower lung levels, which indirectly supported our result. These findings may help to identify targets for localized therapy.

Our study had some potential limitations. First, in our hospital, not all patients with asthma are required to undergo high-resolution CT, and, thus, our findings could not be extrapolated to the entire asthma population. Therefore, further studies, including the full spectrum of patients with asthma, are needed. Second, we tried to analyze the correlations between airway dimension indices and the clinical characteristics of the subjects with asthma. Because regression analysis cannot be performed with repeated measure data, we set clinical characteristics as between-subjects factors to observe differences between the groups. We found that subjects with asthma in the low $\mathrm{FEV}_{1}: \mathrm{FVC}$ (\%) group and the severe group had increased wall area percentage compared with those in the normal $\mathrm{FEV}_{1}: \mathrm{FVC}$ (\%) group and the moderate-mild group. Several studies have reached similar conclusions: airway thickening is related to disease severity and the degree of air-flow obstruction, ${ }^{12,15,18,25,26}$ which, however, differed from the result of Niimi et al ${ }^{12}$; in our study, no significant difference was found among the groups with different disease durations. Possible explanations for this discrepancy could be the following: first, the disease duration might be inconsistent with the disease severity; second, owing to our data type, we could not make an analysis of correlations.

\section{Conclusions}

Our findings indicated that airway remodeling in asthma was more prominent in distal airways and at lower lobe bronchi, which may be helpful to predict small airway involvement and identify targets for localized therapy in asthma.

\section{REFERENCES}

1. Global Initiative for Asthma. Global strategy for asthma management and prevention (2015 update). http://www.ginathma.org. Accessed April 26, 2018.

2. Saetta M, Di Stefano A, Rosina C, Thiene G, Fabbri LM. Quantitative structural analysis of peripheral airways and arteries in sudden fatal asthma. Am Rev Respir Dis 1991;143(1):138-143.

3. Carroll N, Elliot J, Morton A, James A. The structure of large and small airways in nonfatal and fatal asthma. Am Rev Respir Dis 1993;147(2):405-410.

4. Carroll N, Carello S, Cooke C, James A. Airway structure and inflammatory cells in fatal attacks of asthma. Eur Respir J 1996;9(4): 709-715.

5. Bonini M, Usmani OS. The role of the small airways in the pathophysiology of asthma and chronic obstructive pulmonary disease. Ther Adv Respir Dis 2015;9(6):281-293.

6. van den Berge M, Ten Hacken NHT, Cohen J, Douma WR, Postma DS. Small airway disease in asthma and COPD: clinical implications. Chest 2011;139(2):412-423.

7. Kuwano K, Bosken CH, Paré PD, Bai TR, Wiggs BR, Hogg JC. Small airways dimensions in asthma and in chronic obstructive pulmonary disease. Am Rev Respir Dis 1993;148(5):1220-1225.

8. James AL, Paré PD, Hogg JC. The mechanics of airway narrowing in asthma. Am Rev Respir Dis 1989;139(1):242-246.

9. King GG, Paré PD, Seow CY. The mechanics of exaggerated airway narrowing in asthma: the role of smooth muscle. Respir Physiol 1999;118(1):1-13.

10. Al-Muhsen S, Johnson JR, Hamid Q. Remodeling in asthma. J Allergy Clin Immunol 2011;128(3):451-462; quiz 463-464.

11. Lötvall J, Akdis CA, Bacharier LB, Bjermer L, Casale TB, Custovic A, et al. Asthma endotypes: a new approach to classification of disease entities within the asthma syndrome. J Allergy Clin Immunol 2011;127(2):355-360.

12. Niimi A, Matsumoto H, Amitani R, Nakano Y, Mishima M, Minakuchi M, et al. Airway wall thickness in asthma assessed by computed tomography. Relation to clinical indices. Am J Respir Crit Care Med 2000;162(4 Pt 1):1518-1523.

13. Gupta S, Hartley R, Khan UT, Singapuri A, Hargadon B, Monteiro W, et al. Quantitative computed tomography-derived clusters: redefining airway remodeling in asthmatic patients. J Allergy Clin Immunol 2014;133(3):729-738.e18.

14. Asker S, Asker M, Ozbay B. Evaluation of airway wall thickness via high-resolution computed tomography in mild intermittent asthma. Respir Care 2014;59(4):550-556.

15. Little SA, Sproule MW, Cowan MD, Macleod KJ, Robertson M, Love JG, et al. High resolution computed tomographic assessment of airway wall thickness in chronic asthma: reproducibility and relationship with lung function and severity. Thorax 2002;57(3): 247-253.

16. Niimi A, Matsumoto H, Takemura M, Ueda T, Chin K, Mishima M. Relationship of airway wall thickness to airway sensitivity and airway reactivity in asthma. Am J Respir Crit Care Med 2003;168(8): 983-988.

17. Niimi A, Matsumoto H, Takemura M, Ueda T, Nakano Y, Mishima M. Clinical assessment of airway remodeling in asthma: utility of computed tomography. Clin Rev Allergy Immunol 2004;27(1): $45-58$.

18. Aysola RS, Hoffman EA, Gierada D, Wenzel S, Cook-Granroth J, Tarsi J, et al. Airway remodeling measured by multidetector CT is increased in severe asthma and correlates with pathology. Chest 2008;134(6):1183-1191.

19. Gupta S, Siddiqui S, Haldar P, Entwisle JJ, Mawby D, Wardlaw $\mathrm{AJ}$, et al. Quantitative analysis of high-resolution computed to- 


\section{Airway Remodeling in Asthma}

mography scans in severe asthma subphenotypes. Thorax 2010; 65(9):775-781.

20. Ueda T, Niimi A, Matsumoto H, Takemura M, Hirai T, Yamaguchi $\mathrm{M}$, et al. Role of small airways in asthma: investigation using highresolution computed tomography. J Allergy Clin Immunol 2006; 118(5):1019-1025.

21. Edwards RM, Kicska G, Schmidt R, Pipavath SN. Imaging of small airways and emphysema. Clin Chest Med 2015;36(2):335-347, x.

22. Tulic MK, Christodoulopoulos P, Hamid Q. Small airway inflammation in asthma. Respir Res 2001;2(6):333-339.

23. Aysola R, de Lange EE, Castro M, Altes TA. Demonstration of the heterogeneous distribution of asthma in the lungs using CT and hyperpolarized helium-3 MRI. J Magn Reson Imaging 2010;32(6): 1379-1387.

24. Mitsunobu F, Ashida K, Hosaki Y, Tsugeno H, Okamoto M, Nishida $\mathrm{N}$, et al. Decreased computed tomographic lung density during exacerbation of asthma. Eur Respir J 2003;22(1):106-112.

25. Kasahara K, Shiba K, Ozawa T, Okuda K, Adachi M. Correlation between the bronchial subepithelial layer and whole airway wall thickness in patients with asthma. Thorax 2002;57(3):242-246.

26. Awadh N, Müller NL, Park CS, Abboud RT, FitzGerald JM. Airway wall thickness in patients with near fatal asthma and control groups: assessment with high resolution computed tomographic scanning. Thorax 1998;53(4):248-253. 